\title{
Clinical and Paraclinical Findings in Children With Congenital Hepatic Fibrosis: A Single Center 10-Year Study
}

\author{
Seyed Mohsen Dehghani ${ }^{1}$, Amir Saeidi ${ }^{2}$, Farzaneh Nejati ${ }^{2}$, Iraj Shahramian ${ }^{3 *}$, Ali Bazi ${ }^{4}$, Ali Jangjou ${ }^{5}$, Ali \\ Derakhshan $^{2}$, Morteza Salarzaei ${ }^{3}$, Fatemeh Parooie ${ }^{3}$
}

\author{
${ }^{1}$ Shiraz Transplant Research Center, Shiraz University of Medical Sciences, Shiraz, Iran \\ ${ }^{2}$ Pediatric Department, Shiraz University of Medical Sciences, Shiraz, Iran \\ ${ }^{3}$ Pediatric Digestive and Hepatic Diseases Research Center, Amir-Al-Momenin Hospital, Zabol University of Medical \\ Sciences, Zabol, Iran \\ ${ }^{4}$ Faculty of Allied Medical Sciences, Zabol University of Medical Sciences, Zabol, Iran \\ ${ }^{5}$ Emergency Medicine Department, Shiraz University of Medical Sciences, Shiraz, Iran
}

\begin{abstract}
*Corresponding Author: Iraj Shahramian, M.D., Associate Professor, Pediatric Digestive and Hepatic Diseases Research Center, Amir-Al-Momenin Hospital, Zabol University of Medical Sciences, Zabol, Iran. Tel: 054-32232166, Email: ir_buper@yahoo.com
\end{abstract}

Received December 29, 2019; Accepted May 30, 2020; Online Published June 16, 2020

\begin{abstract}
Background: Congenital hepatic fibrosis (CHF) is an autosomal hereditary disorder affecting the porto-biliary system. It is a rare hereditary disorder often presenting in childhood or adolescence with hepatomegaly, splenomegaly, and gastrointestinal bleeding. A timely diagnosis of organomegalies by sonography can prevent esophageal varices. Liver transplantation is now the only cure for CHF.

Objectives: The current study aimed to determine clinical and paraclinical findings in patients diagnosed with CHF from 2008 to 2017.

Methods: This was a descriptive cross-sectional study of all children $<18$ years of age who were diagnosed with CHF from 2008 until 2017 in the pediatric hepatology ward at Nemazee hospital, Shiraz, Iran.

Results: Overall, 32 CHF patients were included during the study period. Of these, $12(37.5 \%)$ and $20(62.5 \%)$ were female and male, respectively. The most frequent clinical presentations at diagnosis were hepatomegaly $(81 \%)$, splenomegaly (68\%), gastrointestinal bleeding $(43 \%)$, abdominal protrusion $(40 \%)$, ascites $(21 \%)$, and epistaxis $(6 \%)$. Severely enlarged livers were observed in 2 patients. Only 5 patients showed a normal-sized spleen, and kidney sonographic findings were normal in 30 patients. Liver enzymes were not severely deviated from the normal range. There was a significant association between spleen size and esophageal varices $(P=0.01)$. Overall, 8 patients were liver transplanted due to decompensated cirrhosis. One patient developed bone marrow suppression secondary to the Epstein bar virus and ultimately succumbed to post-transplant lymphoproliferative disorder. In the study period, 2 girls and 2 boys died of disease complications.

Conclusion: The results of the present study indicated that the most common clinical findings of CHF in pediatric patients are splenomegaly and hepatomegaly presenting as abdominal distention and gastrointestinal bleeding. Laboratory data can be normal in most cases, but ultrasonographic findings (in liver, spleen, and even kidneys) can be helpful. The present study also showed that patients with splenomegaly are at higher risk of esophageal varices.
\end{abstract}

Keywords: Congenital Hepatic Fibrosis, Splenomegaly, Esophageal Varices

\section{Background}

Congenital hepatic fibrosis (CHF) is an autosomal hereditary disorder affecting the porto-biliary system. It is histopathologically characterized by incomplete development of the ductal plate. The ductal plate itself serves as the fetal precursor of biliary system, and its incomplete formation results in misshapen and abnormal intrahepatic vascular ramifications eventually leading to hepatobiliary fibrosis. ${ }^{1}$ Clinical symptoms appear in different ages. Age is widely variable in $\mathrm{CHF}$ patients, ranging from childhood to the fifth or sixth decades of life; however, most patients are diagnosed in adolescence. ${ }^{2-4}$ Patients with CHF can present with abnormal intrahepatic portal venous ramifications and progressive fibrosis of the portal system. These manifestations can be worsened by factors such as alcohol consumption, steatohepatitis, hepatotoxic drugs, and viral

Copyright $\odot 2020$ The Author(s). This is an open-access article distributed under the terms of the Creative Commons Attribution License (http:// creativecommons.org/licenses/by/4.0), which permits unrestricted use, distribution, and reproduction in any medium, provided the original work is properly cited. 
hepatitis. In the clinical setting, the disease manifests with enlarged and misshapen liver. Furthermore, while the left lobe of the liver is palpable under the xiphoid appendix, the right lobe is not evident on clinical examination. In sonographic findings, liver parenchymal echogenicity is seen, and esophageal varices are characteristic secondary to portal hypertension and hypersplenism. ${ }^{1}$

At the molecular level, CHF is, in fact, a hereditary cholangiopathy resulting from mutations in the gene encoding fibrocystin/polyductin (polycystic kidney and hepatic disease 1). PKHD1 (with 66 exons) is one of the longest human genes located at chromosome $6 \mathrm{p} 12$. This gene is translated to a $447 \mathrm{KD}$ protein, the function of which is unknown, localized on the villi and centromeres of biliary ducts and renal tubules. A lack of this protein in the liver triggers small biliary hamartomas, biliary segmental dilatation, and finally portal fibrosis. All of these changes contribute to portal hypertension, which is the main cause of mortality in children with $\mathrm{CHF}^{5,6}$

Four clinical subtypes of CHF include portal hypertension, cholangitis cholestasis, recurrent cholangitis, mixed, and late-presentation disease. ${ }^{7}$ Most patients are diagnosed with no clinical manifestations and have only mild pain in the right upper-abdominal quadrant. Those with portal hypertension may present with esophageal varices and gastrointestinal bleeding. ${ }^{8}$ Hepatomegaly, splenomegaly, and enlarged kidneys may also be seen. ${ }^{9}$ The paraclinical parameters may encompass mild to severe increases in hepatic enzymes, leukopenia, thrombocytopenia, and abnormal renal functional parameters. ${ }^{10} \mathrm{~A}$ definite diagnosis requires liver histopathological examinations. ${ }^{10}$ In biopsy examination, numerous abnormally shaped portal biliary ducts, abnormal ramifications of portal venous, portal fibrosis, fibrotic bridges between portal branches (i.e. portal-portal bridging fibrosis), intra-stromal biliary duct hamartoma (von Meyenburg complexes), and concentrated bile in bile ducts may be seen. ${ }^{1}$ Hepatic fibrosis results from chronic liver damage and the accumulation of extracellular proteins synthesized by myofibroblasts. The myofibroblasts themselves are induced by the action of hepatic stellate cells and portal fibroblasts. ${ }^{11}$

CHF has been associated with a variety of hereditary and non-hereditary conditions such as Caroli syndrome, polycystic kidney disease, Joubert syndrome, Cogan syndrome, Meckel syndrome, Arima syndrome, and Bardet-Biedl syndrome. ${ }^{10}$ In more than half of cases, CHF is associated with polycystic kidney disease. ${ }^{12}$ Around 33\% of the cases are sporadic, while a family history is seen in the rest. ${ }^{13} \mathrm{CHF}$ has been associated with an elevated risk of cholangiocellular carcinoma in $2.5 \%$ to $16 \%$ of cases. ${ }^{14,15}$

\section{Objectives}

There are currently no definite pharmaceutical treatments to reverse histopathological damages in $\mathrm{CHF}$ patients, and the main efforts are devoted to preventing disease complications by administrating anti-fibrotic and endoscopic therapies to implement a shunt. The only definite cure is liver transplantation which is indicated in those with liver failure. An important factor is a timely diagnosis of the condition to prevent its progression. For this purpose, physicians should be aware of the clinical and paraclinical parameters associated with this disease. As there is little evidence on these characteristics, the current study aimed to determine clinical and paraclinical findings in patients diagnosed with CHF from 2008 till 2017.

\section{Methods}

This was a descriptive cross-sectional study that included all children $<18$ years old who were diagnosed with CHF during 2008 until 2017 in the pediatric hepatology ward of Nemazee hospital, affiliated with Shiraz University of Medical Sciences, Shiraz, Iran. Overall, 32 CHF patients were identified during the study period. The inclusion criteria were age at diagnosis $<18$ years and a diagnosis of CHF confirmed through histopathological examination. Data regarding age, gender, clinical presentations, laboratory parameters, ultrasonographic and endoscopic findings were recorded in a checklist using clinical archives.

The gold standard for diagnosis of $\mathrm{CHF}$ is liver histopathology performed by ultrasound-guided percutaneous biopsy or, in some cases, by post-liver transplant biopsy. All of the included patients met the pathological criteria containing (1) dense fibrous septa of different widths, separate liver parenchyma into hepatic islands containing normal vasculature, portal-portal bridging fibrosis, and intact hepatic lobule; (2) a defective remodeling of the ductal plate manifesting as abnormally shaped small bile ducts in the portal area and the cubic or columnar bile duct epithelia forming elongated or cystic cavities; and (3) potentially abnormal changes to the intrahepatic portal vein branches.

The data was then analyzed by (SPSS) version 17, Chicago: SPSS Inc. Means and frequencies were used to describe the data, and the chi-square test, independent samples student $t$ test, and one-way analysis of variance (ANOVA) were used for inferential analyses.

\section{Results}

Thirty-two patients with liver biopsy-verified congenital fibrosis (periportal fibrosis with irregularly shaped proliferating bile ducts) were studied. Of the 32 patients, $12(37.5 \%)$ were female and $20(62.5 \%)$ were male. In the study period, 2 girls and 2 boys died of the disease. The mean age was $5.6 \pm 4.1$ with a range of 6 months to 16 years old.

The most frequent clinical presentations at diagnosis were hepatomegaly (81\%), splenomegaly (68\%), gastrointestinal bleeding (43\%), abdominal protrusion (40\%), ascites (21\%), and epistaxis (6\%). The laboratory features at diagnosis have been noted in Table 1.

Ultrasonography of the liver, kidney, and spleen was performed for all patients. Severely enlarged livers were observed in 2 patients (Table 2). Only 5 patients showed normal size spleen, and kidney ultrasonographic findings 
Table 1. Laboratory Findings in 32 Children With CHF at Presentation

\begin{tabular}{|c|c|c|c|c|}
\hline Parameters & Mean \pm SD & Min & Max & Median \\
\hline Hemoglobin (g/dL) & $10 \pm 1.6$ & 6.2 & 12.8 & 10 \\
\hline Platelet $\left(10^{3} / \mu \mathrm{L}\right)$ & $145 \pm 71$ & 18000 & 270000 & 13200 \\
\hline White blood cell $\left(10^{3} / \mu \mathrm{L}\right)$ & $5400 \pm 2900$ & 470 & 15300 & 4750 \\
\hline Prothrombin time (s) & $15.3 \pm 6.6$ & 13 & 44 & 13.3 \\
\hline International normalized ratio & $1.63 \pm 1.28$ & 1 & 8 & 1.18 \\
\hline Aspartate aminotransferase (IU/L) & $54 \pm 52.4$ & 3 & 230 & 41 \\
\hline Alanine amino transferase (IU/L) & $50.6 \pm 32.3$ & 9 & 143 & 39 \\
\hline Alkaline phosphatase (IU/L) & $512.8 \pm 375.5$ & 85 & 1700 & 411.5 \\
\hline Albumin $(\mathrm{g} / \mathrm{dL})$ & $3.9 \pm 0.6$ & 2.6 & 5 & 4 \\
\hline Total bilirubin (mg/dL) & $1.4 \pm 1.24$ & 0.1 & 6 & 1 \\
\hline Blood urea nitrogen (mg/dL) & $17.2 \pm 15.6$ & 0.8 & 90 & 14.5 \\
\hline Creatinine (mg/dL) & $0.7 \pm 0.46$ & 0.1 & 2.2 & 0.8 \\
\hline
\end{tabular}

were normal in 30 patients (Table 3 ). No associations were found between spleen size and white blood cell count, platelet count, or hemoglobin level.

In endoscopic findings, esophageal varices with grades 1 , 2,3 , and 4 were observed in 6, 3,2, and 1 patients, respectively. One patient showed grades 2 and 3 simultaneously. Another patient also showed concomitant grades 3 and 4 of the disease. There was a significant association between spleen size and esophageal varices $(P=0.01)$. Overall, 8 patients were liver transplanted due to decompensated cirrhosis, while none of them received liver grafts from a family member. Whole organ was transplanted in 4 patients while the other 4 patients received partial grafts. One patient developed bone marrow suppression secondary to the Epstein bar virus and ultimately succumbed to post-transplant lymphoproliferative disorder. Two of the

Table 2. Liver Ultrasonography Findings in 32 Children With CHF

\begin{tabular}{lll}
\hline Parameters & & No. $\mathbf{( \% )}$ \\
\hline \multirow{3}{*}{ Size } & Normal & $3(9.4)$ \\
& Mild increase & $18(56.2)$ \\
& Moderate increase & $9(28.1)$ \\
& Severe increase & $2(6.3)$ \\
Border & Regular & $20(62.5)$ \\
& Irregular & $12(37.5)$ \\
Echogenicity & Normal & $8(25)$ \\
Portal hypertension & Increased & $24(75)$ \\
Mass & & $12(37.5)$ \\
\hline
\end{tabular}

Table 3. Spleen and Kidney Ultrasonography Findings in 32 Children With $\mathrm{CHF}$

\begin{tabular}{|c|c|c|c|}
\hline Parameters & & & No. $(\%)$ \\
\hline \multirow{3}{*}{ Spleen size } & & Normal & $5(15.6)$ \\
\hline & & Mild to moderate & $19(59.4)$ \\
\hline & & Severe increase & $8(25)$ \\
\hline \multirow{5}{*}{ Kidney } & \multirow{3}{*}{ Size } & Normal & $30(93.7)$ \\
\hline & & Decreased & $0(0)$ \\
\hline & & Increased & $2(6.3)$ \\
\hline & \multirow{2}{*}{ Echo } & Normal & $22(68.7)$ \\
\hline & & Increased & $10(31.3)$ \\
\hline
\end{tabular}

patients were kidney transplanted due to end stage renal disease secondary to associated polycystic kidney, and one of them remained hemodialysis-dependent due to graft rejection.

\section{Discussion}

Out of the 32 studied patients, only 3 had normal livers and 5 had normal spleens; however, the majority had normalsized kidneys. Endoscopy showed esophagus varices of variable grades in 29 patients. Liver transplantation was performed in 8 patients. No significant associations were found between WBC $(P=0.43)$, platelet count $(P=0.21)$, or hemoglobin $(P=0.17)$ and spleen size. Furthermore, there were no relationships between platelet count $(P=0.53)$, liver enzymes $(P=0.11)$, or age $(P=0.28)$ and the presence of esophageal varices. Nonetheless, a significant correlation was noted between spleen size and esophageal varices. The mean patient age was $5.6 \pm 4.1$ years with half of them aged $<6$ years old, which was similar to a report in Spain. In a recent study, the age range of patients presenting with $\mathrm{CHF}$ symptoms was 1 to 15 years old. ${ }^{1,16}$ The diagnosis age ranged from 7.5-28.4 years in studies conducted in India, ${ }^{17}$ Saudi Arabia, ${ }^{9}$ Pakistan, ${ }^{18}$ and Turkey. ${ }^{10}$ The mean age was higher in these precedent studies respective to the current report. This was due to the inclusion of patients $<18$ years of age in this study, rendering a lower mean age. Liver enzymes were normal or mildly increased in patients of the current study. Mildly elevated liver enzymes were noted in some patients in a US study on CHF. ${ }^{19}$ In two other studies in Spain ${ }^{16}$ and Pakistan, ${ }^{18}$ the liver enzymes of CHF patients were in the normal range. In the current study, it was noted that patients showed elevated alkaline phosphatase levels, which was in line with a study in Saudi Arabia. ${ }^{9}$ Ultrasonography findings further showed increased liver echogenicity, increased liver size, and irregular liver borders in $75 \%$, $90.4 \%$, and $37.5 \%$ of patients, respectively. In a study in the United States, 92\%, 41\%, and $61 \%$ of CHF patients had increased echogenicity, size, and irregular borders, respectively. ${ }^{19}$ Likewise, all 25 children with $\mathrm{CHF}$ had increased liver sizes in a study in Pakistan. ${ }^{18}$ Furthermore, only 5 out of the 32 patients in the current study had a normal-sized spleen; the rest (84\%) had splenomegaly in 
ultrasonographic findings. Increased spleen size has been reported in $92 \%$ and $96 \%$ of $\mathrm{CHF}$ patients in studies in the United States ${ }^{19}$ and Pakistan, ${ }^{18}$ respectively. This study detected no association between either platelet count or other clinical variables and spleen size, which is opposite of a report in the United States which noted a significant association between platelet count and spleen dimensions in CHF patients. ${ }^{19}$ In endoscopic observations in the current study, esophageal varices were identified in $81 \%$ of patients. Esophageal varices were reported in $71 \%, 66 \%$, and $100 \%$ of CHF patients in studies in the United States, ${ }^{19}$ Tunisia, ${ }^{20}$ and Saudi Arabia. ${ }^{9}$ A significant relationship was found between spleen size and the presence of esophageal varices in the current study. Considering the invasive nature of endoscopy, this is an important finding which can guide physicians to perform this procedure in $\mathrm{CHF}$ patients with splenomegaly. The detection of esophageal varices is an important clinical factor in following up with patients and prognoses.

The most frequent clinical presentations in the current patients were hepatomegaly and splenomegaly, respectively. Splenomegaly was also noted as the most frequent clinical findings in CHF patients in a study performed in the United States. ${ }^{19}$ In a similar report in Turkey, abdominal distention due to splenomegaly and hepatomegaly was reported to be the most common finding, while hematemesis was noted as the least frequent. ${ }^{10}$ In another report from Saudi Arabia, growth retardation was described as the most common finding followed by gastrointestinal bleedings including hematemesis and melena. ${ }^{9}$ In line with the current observations, hematemesis and splenomegaly were noted as the most common clinical findings in CHF patients in two studies in Spain ${ }^{16}$ and Pakistan. ${ }^{18}$ Different presenting complications can be related to different disease stages secondary to delayed diagnosis in some patients. Being aware of the disease and its features can help physicians early diagnose the condition before progression of the disease and the emergence of hemorrhagic sequel.

\section{Conclusion}

The results of the present study indicated that the most common clinical findings of $\mathrm{CHF}$ in pediatric patients are splenomegaly and hepatomegaly presenting as abdominal distention and gastrointestinal bleeding. Laboratory data can be normal in most cases but ultrasonographic findings (in liver, spleen, and even kidneys) can be helpful. The present study also showed that patients with splenomegaly are at higher risk of esophageal varices.

\section{Authors' Contributions}

$\mathrm{SMD}, \mathrm{AS}, \mathrm{FN}$ and IS participated in conception and design of the study, library searches and assembling relevant literature, critical review of the paper, supervising writing of the paper and database management. $\mathrm{AB}, \mathrm{AJ}, \mathrm{AD}, \mathrm{MS}$ and FP participated in data collection, library searches and assembling relevant literature, writing the paper, critical review of the paper and analysis of the data and critical

\section{Research Highlights}

\section{What Is Already Known?}

Splenomegaly and hepatomegaly are reported to be the most common clinical findings of $\mathrm{CHF}$ in pediatric patients who are often referred with abdominal distention and, sometimes, gastrointestinal bleeding.

\section{What This Study Adds?}

Although sonography can be quite helpful, laboratory data can be normal in most cases. Splenomegaly was showed to be associated with a higher risk of esophageal varices. A significant relationship was found between spleen size and the presence of esophageal varices in the current study. Considering the invasive nature of endoscopy, this is an important finding which can guide physicians to perform this procedure in CHF patients with splenomegaly. The detection of esophageal varices is an important clinical factor in following up with patients and prognoses.

review of the paper. All authors read and approved the final manuscript.

\section{Conflict of Interest Disclosures}

The authors declare that they have no conflicts of interest.

\section{Ethical Approval}

Informed consent was obtained from children's parents.

\section{Acknowledgments}

We are grateful to the parents and patients who participated in this study.

\section{References}

1. Irie R, Nakazawa A, Sakamoto S, et al. Living donor liver transplantation for congenital hepatic fibrosis in children. Pathol Int. 2020;70(6):348-354. doi:10.1111/pin.12917.

2. Zhu B, Du Z, Wang Z, Li Y, Zhang J, Zhu H. Congenital hepatic fibrosis in children and adults: clinical manifestations, management, and outcome-case series and literature review. Gastroenterol Res Pract. 2020;2020:8284274. doi:10.1155/2020/8284274

3. Shrestha A, Al-Mahtab M, Rahman S, Sarkar J, Lama TK. Its congenital hepatic fibrosis; not cirrhosis at all. Nepal Med J. 2018;1(2):124-126. doi:10.3126/nmj.v1i2.21624.

4. Wu X, Du XR, Ding JF, Wu MJ, Luo SQ, Feng XZ. Clinical features of congenital hepatic fibrosis in children. J Clin Pediatr. 2016;34(6):444-448. doi:10.3969/j.issn.10003606.2016.06.011.

5. Locatelli L, Cadamuro M, Spirlì C, et al. Macrophage recruitment by fibrocystin-defective biliary epithelial cells promotes portal fibrosis in congenital hepatic fibrosis. Hepatology. 2016;63(3):965-982. doi:10.1002/hep.28382.

6. Rock N, Kanavaki I, McLin V. Congenital hepatic fibrosis, Caroli's disease, and other fibrocystic liver diseases. In: Guandalini S, Dhawan A, Branski D, eds. Textbook of Pediatric Gastroenterology, Hepatology and Nutrition: A Comprehensive Guide to Practice. Cham: Springer; 2016. p. 647-661. doi:10.1007/978-3-319-17169-2_57. 
7. Alsomali MI, Yearsley MM, Levin DM, Chen W. Diagnosis of congenital hepatic fibrosis in adulthood. Am J Clin Pathol. 2020;153(1):119-125. doi:10.1093/ajcp/aqz140.

8. Yönem O, Ozkayar N, Balkanci F, et al. Is congenital hepatic fibrosis a pure liver disease? Am J Gastroenterol. 2006;101(6): 1253-1259. doi:10.1111/j.1572-0241.2006.00642.x.

9. Guerra JA, Kampa KC, Zapparoli M, Alves VA, Ivantes CAP. Congenital hepatic fibrosis and obliterative portal venopathy without portal hypertension - a review of literature based on an asymptomatic case. Arq Gastroenterol. 2018;55(4):324-328. doi:10.1590/s0004-2803.201800000-91.

10. Shorbagi A, Bayraktar Y. Experience of a single center with congenital hepatic fibrosis: a review of the literature. World J Gastroenterol. 2010;16(6):683-690. doi:10.3748/wjg.v16. i6.683.

11. Gäbele E, Brenner DA, Rippe RA. Liver fibrosis: signals leading to the amplification of the fibrogenic hepatic stellate cell. Front Biosci. 2003;8:d69-77. doi:10.2741/887.

12. Jiang L, Fang P, Weemhoff JL, Apte U, Pritchard MT. Evidence for a "Pathogenic Triumvirate" in congenital hepatic fibrosis in autosomal recessive polycystic kidney disease. Biomed Res Int. 2016;2016:4918798. doi:10.1155/2016/4918798.

13. Al Sarkhy A, Hassan S, Alasmi M, Assiri AM, Alkuraya FS. Congenital hepatic fibrosis in a child with Prader-Willi syndrome: a novel association. Ann Saudi Med. 2014;34(1):8183. doi:10.5144/0256-4947.2014.81.
14. Janowski K, Goliszek M, Cielecka-Kuszyk J, Jankowska I, Pawłowska J. Congenital hepatic fibrosis in a 9-year-old female patient - a case report. Clin Exp Hepatol. 2017;3(3):176-179. doi:10.5114/ceh.2017.70299.

15. Patel JN, Gupta S, Fauzdar M, Patel N, Chaturvedi S. Congenital hepatic fibrosis associated with polycystic kidney disease. J Liver. 2015;4(1):171. doi:10.4172/2167-0889.1000171.

16. Ramírez Mayans JA, Mata Rivera N, Mora Tiscareño MA, et al. [Congenital hepatic fibrosis. Study of 26 cases]. Acta Gastroenterol Latinoam. 1994;25(5):297-303.

17. Poddar U, Thapa BR, Vashishta RK, Girish CS, Singh K. Congenital hepatic fibrosis in Indian children. J Gastroenterol Hepatol. 1999;14(12):1192-1196. doi:10.1046/j.14401746.1999.02028.x.

18. Parkash A, Cheema HA, Malik HS, Fayyaz Z. Congenital hepatic fibrosis: clinical presentation, laboratory features and management at a tertiary care hospital of Lahore. J Pak Med Assoc. 2016;66(8):984-988.

19. Gunay-Aygun M, Font-Montgomery E, Lukose L, et al. Characteristics of congenital hepatic fibrosis in a large cohort of patients with autosomal recessive polycystic kidney disease. Gastroenterology. 2013;144(1):112-121.e112. doi:10.1053/j. gastro.2012.09.056.

20. Mazigh MS, Aloui N, Fetni I, et al. [Congenital hepatic fibrosis in children. Report of 9 cases and review of the literature]. Tunis Med. 2006;84(3):182-188. 